\title{
Improving Language Containment Using Fairness Graphs
}

\author{
Ramin Hojati ${ }^{1}$ (UC Berkeley) \\ Robert Mueller-Thuns (Cadence Design Systems) \\ Robert K. Brayton (UC Berkeley)
}

\begin{abstract}
Language containment is one important approach to formal design verification. When working at a higher, more abstract level, additional unwanted behavior may be introduced in the model that can be excluded for the verification step using so-called faimess constraints. The language containment computation using Binary Decision Diagrams (BDD's) typically involves performing reachability analysis, early failure detection, and then applying a set of operators until convergence is achieved ([HTKB92]). The running time of the latter part (called the main computation) is correlated with the number of faimess constraints. In this paper, we introduce techniques which improve the efficiency of the main computation by analyzing a graph induced by the faimess constraints. This graph can be built efficiently using BDD's. We have implemented our algorithms in the verification system HSIS, and experimental results demonstrate the effectiveness of these ideas.
\end{abstract}

\section{Introduction}

Design Verification is the process of answering the question "Is what I specified what I wanted?" This is accomplished by specifying the system at a suitable level of abstraction and then proving properties of the system. For example, in a large design one can abstract the design to functional blocks and verify that the communication between blocks is correct. An example of a property may be that no two functional units write to the global bus at the same time, a so-called safety property.

The most widely used scheme for modeling a system is to use a set of interacting non-deterministic finite state machines. Equivalently, the system can be represented by its product machine. Recently, Binary Decision Diagrams (BDD's) ([Bry86]) have been used for representing FSM's. Using this scheme, the transition relation of each machine is represented by a BDD. Let $T_{j}\left(x_{j}, i_{j}, y_{j}\right)$ be the transition relation of the $j$-th FSM, where $x_{j}$ represents the present state variable of the machine, $y_{j}$ the next state, and $i_{j}$ the set of inputs and outputs of the machine. We generally assume that the system is closed, so all inputs to a machine are produced by some other machine. The product machine is then represented by $T(x, i, y)=\prod_{j} T_{j}\left(x_{j}, i_{j}, y_{j}\right), x$ and $y$ are the set of present and next state variables, and $i$ is the rest of the variables (referred to as the $i$ ) $o$ variables).

Abstraction may result in unwanted behaviors. Modeling unbounded but finite delays is an example. Here we want to allow the system to stay at a state $s$ for some finite but unknown amount of time. The behaviors where the system is in $s$ forever should be excluded. An easy abstraction is to allow infinite delays, but then all properties of the system should only be checked on traces where the system gets out of $s$ infi-

1. The first author is supported by the Semiconductor Research Corporation under grant 94-DC-008. 
nitely often if it enters $s$ infinitely often. A second example of unwanted behavior is the modeling of schedulers. Assume that a set of processes are executed in a system controlled by a fair scheduler; one which disallows starvation of processes. An example of a fair scheduler is a round robin scheduler. To avoid having to model the details of a particular scheduler, system constraints can be used to disallow looking at traces where one process is blocked forever. Such constraints on a system's traces are called fairness constraints (FCs). In our environment, abstraction and fairness constraints are allowed. Hence, for us a hardware system consists of a model of the hardware plus a set of fairness constraints.

Having specified the system, the next step is the verification of properties. For verification, one can use Computation Tree Logic (CTL) ([CES86]) formulas, or $\omega$-automata ([VW86], [Kur87]). In this paper, we are concerned only with $\omega$-automata verification. To verify a property expressed using $\omega$-automata, we use language containment $(L C)$, which is the process of verifying that the language of a system is contained in the language of a property automaton. Language containment is performed by checking language emptiness of the intersection of the complement of the language of the property automaton and the language of the system.

The most general language containment environment used in practice is the edgeStreettledge-Rabin (eSeR) environment, where the system is specified using edgeStreett automata and the property using edge-Rabin automata. The techniques we present in this paper are designed for the L-environment ([Kur87]), which is a subset of eSeR. It is shown in [HSB94] that the L-environment and eSeR have the same expressive power, however, eSeR can be (exponentially) more compact. The extension of our techniques to eSeR remains an open question.

To check for language containment, the product of the system and the complement of the property is built. The language containment check reduces to checking whether the language of this automaton is empty, which in turn is equivalent to answering the question of whether there is any run (i.e. an infinite path) satisfying all fairness constraints. Such runs are called fair runs. To do so, first the set of reachable states is computed, and the active set is initialized to this set. A technique known as early failure detection, which find easy failures quickly, is applied next. If no errors are found, then a subset of reachable states which cannot contain any fair runs is deleted from the active set. The last step is to apply a set of operators which trim portions of the current active set which cannot possibly contain any fair runs. We refer to this part of the computation as the main computation. The time-consuming operators in this set are known as fair-path operators, which perform a reachability computation for each fairness constraint. Hence, the running-time of the main computation is correlated with the number of fairness constraints.

Our hope is to make language containment with faimess constraints essentially as efficient as language containment without fairness constraints. Since in verification the reachable set is computed, and since early failure detection is relatively cheap, we need to speed up the last part of the algorithm to achieve our goal. In some cases, this last part is a significant portion of the running-time of the algorithm.

In this paper, we propose to take advantage of a graph induced by the fairness constraints, known as the fairness graph. If this graph is acyclic, then there are no fair runs. Hence, the main computation can be bypassed. If it contains cycles, then in some situations we can combine set of faimess constraints, and hence reduce the number of fairness constraints in the main computation. The problem of minimizing the number of faimess constraints at this step reduces to the partition into forest problem, 
which is NP-complete (GJ79]). So we use a greedy heuristic for it. We have implemented our techniques in the formal verification HSIS using BDD's. Based on limited experience, we observed that our techniques can reduce the run-time of the main computation when it is significant.

The paper's flow is as follows. Section 2 defines the preliminaries. Section 3 defines the fairness graph and states its properties. Section 4 presents our BDD based method for building the fairness graph. Section 5 formulates the optimization problem associated with clustering the cycle sets, states its NP-completeness, and describes our heuristic. Section 6 presents some experimental results. Conclusions and future work are given in section 7 .

\section{Preliminaries}

Definition A Finite State Machine (FSM) or Automaton $A$ is a tuple $(\Sigma, Q, T, I)$, where $\Sigma$ is a finite alphabet, $Q$ is a finite set of states, $T$ is a transition relation on $Q \times \Sigma \times Q$, and $I$ is a set of initial states. A run $r$ of an $\omega$-string $x$ in $A$ is an infinite sequence of states, such that the first state $r_{0} \in I$, and for all $i,\left(r_{i}, x_{i}, r_{i+1}\right) \in T$. The set of states occurring infinitely often in a run $r$, called the infinitary set, is denoted by $\inf (r)$. Since the set of states $Q$ is finite, $\inf (r)$ is always a non-empty finite set. The set of all $\omega$-strings which have a run in $A$ is called the behavior or language of $A$.

It is routine to represent FSM's and sets of states using BDD's. We refer the reader to one such reference [Tou90].

In practice, one models a system using a set of FSM's. The language of the system, or the behavior of the system, is the intersection of the languages of all component FSM's, which can be represented using the product machine. Because of abstraction, one may add some unwanted behavior, which can be excluded using faimess constraints. In the L-environment, one uses L-processes for this task.

Definition An $L$-process is a FSM with a set of fairness constraints, which are a set of edges, known as recur edges, and a set of subsets of states, known as cycle sets. A string $x$ is accepted if there exists a run of $x$, such that no recur edges is traversed infinitely often, and $\inf (r)$ is not contained in any cycle set.

Consider the following example. Assume a FSM can get into a state $s$, in which it stays for 100 clock ticks. One might model this by putting a self-loop on $s$ (note that the FSM can get out of state $s$ non-deterministically at any point). This will reduce the state space, since otherwise 100 different states for $s$ are needed. However, the problem is now that the FSM can stay in $s$ forever. This problem is known as modeling indefinite but finite delay. To model this, we can use a cycle set. By putting a cycle set around $s$, we exclude the behavior that the FSM stays in $s$ forever.

Hence, a system is specified as a set of interacting FSM's, each having its own set of faimess constraints. A behavior is legal if it is a fair behavior (one satisfying the fairness constraints) in every machine. The product L-process is the product FSM, with the faimess constraints of each FSM lifted to the product machine. Lifting means the following. Assume a cycle set is given. Each cycle set is a set $S$ of states of a FSM $P_{i}$. The cycle set is changed to the set $S^{\prime}$ of those states of the product machine whose projection onto $P_{i}$ is $S$. When BDD's are used to represent sets of states, lifting a set of states from a component machine to the product machine, is just thinking of the BDD as being defined over the set of variables of all components. Hence, no computations need to be done. It turns out that this product L-process accepts the intersection of the languages of the component L-processes. 
To describe a property, one can use an L-automaton.

Definition An L-automaton is defined syntactically the same as an L-process, i.e. it is a finite automaton whose acceptance conditions are described by a set of recur edges and cycle sets. However, the interpretation is complementary. A string $x$ is accepted by an L-automaton if there exists a run $r$ of $x$ such that either some recur edge is traversed infinitely often, or $\inf (r)$ is contained in some cycle set.

The L-environment can be summarized as follows.

1. Express the system using a set of L-processes, or equivalently by their product Lprocess.

2. Describe the property using a strongly deterministic and complete L-automaton. A complete automaton is one which has a transition for every symbol from every state.

3. If we think of the deterministic L-automaton as an L-process, the language is complemented. So, it remains to check that the intersection of the languages of the product L-process describing the system and the L-process obtained from the property Lautomaton is empty. This intersection can be represented using another L-process.

We have therefore reduced our task to checking that the $L(P)=\varnothing$, for some L-process $P$ with cycle sets $c_{1}, \ldots, c_{n}$ and recur edges $\operatorname{Rec}(x)$. To do this check, we compute the set of reachable states, and restrict the transition relation of the L-process to the set of reachable states. We then remove the recur edges. Call this machine $\tilde{P}$. Now, $L(P)=\varnothing$ iff no cycle of $\tilde{P}$ is contained in any of the cycle sets. We call such cycles fair cycles.

To check for the existence of fair cycles, we first check whether there are any cycles involving states in $\Gamma$, where $\Gamma=\bigcap_{j} \vec{c}_{j}$. Note that any such cycle is fair. If there are no such cycles, we delete all states in $\Gamma$ from the set of reachable states and $\tilde{P}$. Call this machine $Q$. To check for fair cycles in $Q$, we apply a set of operators (which we will define below) until convergence is achieved. The set converged to is not empty iff there exists a fair cycle.

In what follows, let $G$ be a graph, $V$ the set of its vertices, $A \subseteq V$ a subset of its vertices, and $T(x, y)$ the transition (edge) relation of $G$.

Definition Let $F$ be a monotone increasing $k$-ary predicate transformer. Define the least fixpoint of $F$ given $Q$, denoted by $\mu(X, Q) . F X$ where $Q$ is an $k$-ary over sets $D_{1}, \ldots, D_{k}$, by the set $F^{i}(Q)$ such that $F\left(F^{i}(Q)\right)=F^{i}(Q)$. Intuitively, $X$ is the variable we are recurring on, and $Q$ is its initial value. Similarly, define the greatest fixpoint of a monotone decreasing $k$-ary predicate transformer $F$ given $Q$, denoted by $\vee(X, Q) . F X$, by the set $F^{i}(Q)$ such that $F\left(F^{i}(Q)\right)=F^{i}(Q)$.

Definition Let $S_{1}(A, y)=\exists x(A(x) \wedge T(x, y))$ and $S_{1}(x, A)=\exists y(A(y) \wedge T(x, y))$. Thus, $S_{1}(A, y)$ are the successors of $A(x)$ and $S_{1}(x, A)$ are the predecessors of $A(x)$.

Definition Let $R_{1}(A, y)=A(y) \vee S_{1}(A, y), \quad R_{1}(x, A)=A(x) \vee S_{1}(x, A)$, $R^{*}(A, y)=\mu(X, A) \cdot\left(R_{1}(X, y)\right), R^{*}(x, A)=\mu(X, A) \cdot\left(R_{1}(x, X)\right)$. Note that the first two are "one-step" operators, while the last two compute the least fixed-point containing $A$. One should read $R^{*}(A, y)$ as the set of points reachable from $A$. Similarly 
$R^{*}(x, A)$ is the set of points that can reach $A . R_{1}(A, y)$ and $R_{1}(x, A)$ are the one-step versions of these, respectively.

Definition We define two "stable set" operators, $S^{*}(A, y)=v(X, A) \cdot\left(S_{1}(X, y)\right)$ and $S^{*}(x, A)=v(X, A) \cdot\left(S_{1}(x, X)\right)$. One can think of the first as calculating the backward stable set contained in $A$ (i.e. the set of all vertices which are reached by some vertex involved in some cycle in $A$ ), and the latter as the forward stable set of $A$ (i.e. the set of all vertices which can reach some vertex involved in some cycle in $A$ ).

Definition Define the forward fair-path operator by $F(x)=\prod_{j} R^{*}\left(x, \bar{c}_{j} \wedge A\right)$, where $A$ is the current active set. Note that if a state can reach a fair cycle, it is not deleted by this operator. Similarly, define the backward fair-path operator by $B(x)=\prod_{j} R^{*}\left(\vec{c}_{j} \wedge V, y\right)$. Note, if a state is reached by a fair cycle, it is not deleted by this operator.

One method for checking language containment is to apply forward and backward stable set, and forward and backward fair-path operators. If we converge to a nonempty set, then $L(P)=\varnothing$. Otherwise, it is not. The time-consuming part of this computation is the application of the fair-path operators. Each fair-path operator performs a reachability computation for each cycle set. Hence, we expect its runningtime to be correlated strongly with the number of cycle sets. In the next few sections, we use the properties of a graph induced by the cycle sets in $Q$ to reduce the run-time of this portion of the computation.

\section{The Fairness Graph and Its Properties}

In what follows, we assume $Q$ is the state graph of an automaton with cycle sets $c_{1}, \ldots, c_{n}$, such that every node is contained in at least one cycle set (which is the case after early failure detection).

Definition The fairness graph $Q_{f}$ induced by the cycle sets is a graph on $n$ nodes $\tilde{c}_{1}, \ldots, \tilde{c}_{n}$ which has an edge from vertex $\tilde{c}_{i}$ to $\tilde{c}_{j}$ iff there exists an edge $(u, v)$ in $Q$ such that $v \in c_{i}, w \in c_{j}$, and $w \notin c_{i}$.
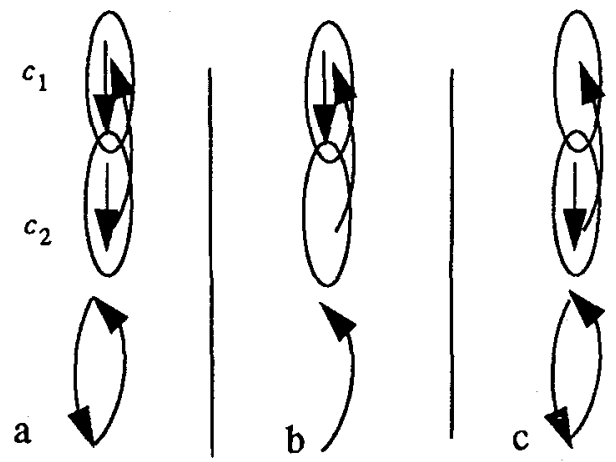

\section{Figure 1}

Examples In the above figure three examples of graphs and their faimess graphs are given. The original graphs are on top, and the faimess graphs are at bottom. Each oval around the vertices signifies a cycle set. Note that in example $b$, there is no edge from 
the first cycle set to the second one, since there is no edge from $c_{1}$ to $c_{2}$ whose endpoint is not in $c_{1}$. Also, note that in example $c$, although there is no cycle in the original graph, there is a cycle in the fairness graph.

Definition Let a path in $Q x_{1}, \ldots, x_{p}$ be given. Let $c_{1}, \ldots, c_{p}$ be a sequence of cycle sets such that for all $i, x_{i} \in c_{i}$. We call this sequence a projection of a path. Let a shadow of a path be obtained from a projection of a path by deleting consecutive duplicate copies of a cycle set.

Intuitively, as we follow a path we go through a sequence of cycle sets. A shadow of a path is obtained by only counting those cycle sets which are different than the current one. Note that the shadow of a path is a sequence of states in its faimess graph. The following figure gives an example of a path and its shadows, which shows that the shadow of a path may not be unique, since the projection of a path may not be unique.
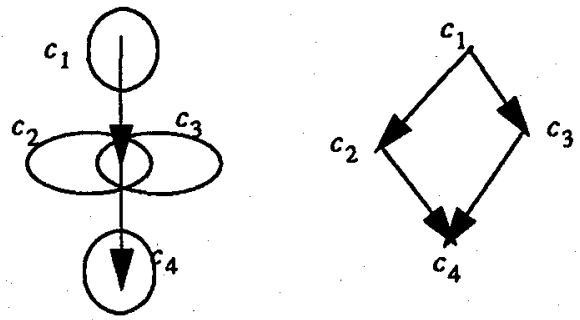

\section{Figure 2}

We have the following properties for $Q_{f}$, the fairness graph. The proofs of those lemmas which follow directly from the definition of the faimess graph are not given.

Lemma 1 There are no self-loops in $Q_{f}$.

Lemma 2 Let $\Gamma$ be a cycle, a shadow of which visits $c_{i_{1}}, \ldots, c_{i_{i}}, c_{i_{1}}$ in order. Then, there is a corresponding cycle $\tilde{c}_{i_{1}}, \ldots, \tilde{c}_{i_{k}}, \tilde{c}_{i_{1}}$ in $Q_{f}$.

Lemma 3 Every shadow of a fair cycle is a cycle in $Q_{f}$ containing more than one state (recall that a fair cycle is a cycle not contained in any of the cycle sets).

Lemma 4 There may be a cycle $\tilde{c}_{i_{1}}, \ldots, \tilde{c}_{i_{k}}, \tilde{c}_{i_{1}}$ in $Q_{f}$, but no corresponding cycle in $Q$ that visits $c_{i_{1}}, \ldots, c_{i_{k}}, c_{i_{1}}$.

Proof In the above figure, $c$ is an example.

Theorem 1 Let $D$ denote the set of all vertices in $Q_{f}$ which are not contained in any cycle. Let $Q^{\prime} \subseteq Q$ be a new graph where all the vertices which are contained in some cycle set in $D$ have been deleted. The cycle sets of $Q^{\prime}$ are the same cycle sets of $Q$ except for those contained in $D$. Then, every fair cycle of $Q$ is present in $Q^{\prime}$ (note that every fair cycle of $Q^{\prime}$ is clearly a cycle set in $Q$ ).

Proof It suffices to show that every state involved in some fair cycle in $Q$ is present in $Q^{\prime}$. Let $x \in Q$ be involved in some fair cycle. Every fair cycle of $Q$ has a shadow which is a cycle in $Q_{f}$. Hence, all cycle sets containing $x$ will not be deleted by the above procedure $(\mathrm{QED})$.

Note that the above procedure gives us a method for deleting portions of the active set in which no fair behavior can exist. In some cases, as the following corollary shows, we may be able to decide the emptiness check without any further computations.

Corollary 1 If $Q_{f}$ is acyclic, then there are no fair cycles in $Q$. 
Corollary 1 If $Q_{f}$ is acyclic, then there are no fair cycles in $Q$.

\section{Building the Fairness Graph}

Building the fairness graph is the most time-consuming part of the overhead of our algorithm. Note that if we were not using BDD's, building this graph would have been very time-consuming, since possibly all edges in the product $L$-process had to be visited. There are many ways to build this graph using BDD's, some performing better than others. In section 4.1, we first present a naive algorithm, and then present one which improves the performance of the first one. In section 4.2 , we discuss ways of reflecting the structure of $Q$ better in $Q_{f}$, by deleting states which cannot contain any fair cycles.

\subsection{Algorithms}

To build the faimess graph using BDD's, we introduce two new variables which take values from 1 to $n$. The first variable, denoted by $x^{\prime}$, corresponds to the present state of the faimess graph, whereas the second one, denoted by $y^{\prime}$, corresponds to the next state. We eventually build a transition relation $R\left(x^{\prime}, y^{\prime}\right)$, which represents the fairness graph. Let $A(x)$ represent the active set of states, which is originally the set of reachable states with the states in $\bigcap_{i} \bar{c}_{i}$ deleted. We assume the transition relation of $Q$, $T(x, y)$, is restricted to $A(x)$. The following lemma provides a straight-forward way to build the faimess graph.

Lemma $5 \sum_{i} \sum_{j} \exists x \exists y\left(T(x, y) \wedge c_{i}(x) \wedge c_{j}(y) \wedge \overline{c_{i}(y)} \wedge\left(x^{\prime}=i\right) \wedge\left(y^{\prime}=j\right)\right)$ computes $Q_{f}$. This computation does $2 n^{2}$ quantifications, $5 n^{2}$ Boolean AND's, and $n^{2}$ Boolean OR's.

Proof For every pair of cycle sets $c_{i}$ and $c_{j}$ the above computation asks whether there is an edge between them. If so, an edge $(i, j)$ is added to the final relation. Hence, the relation represents $Q_{f}$. The number of operations follows by expanding the above sums, which has exactly $n^{2}$ terms (QED).

The improved version involves re-arranging the order of various operations.

Lemma $6 \quad \sum_{j} \exists y\left(\sum_{i}\left(\exists x\left(T(x, y) \wedge c_{i}(x)\right)\right) \wedge \overline{c_{i}(y)} \wedge\left(x^{\prime}=i\right)\right) \wedge c_{j}(y) \wedge\left(y^{\prime}=j\right)$

computes $Q_{f}$. Moreover, it takes $2 n$ quantifications, $5 n$ Boolean AND's, and $2 n$ Boolean OR's.

Proof It suffices to show that the above equation is algebraically equal to the one in the previous lemma. This follows by noticing that existential quantification and Boolean sums commute. To count the number of operations, note that $\sum_{i}\left(\exists x\left(T(x, y) \wedge c_{i}(x)\right)\right) \wedge \overline{c_{i}(y)} \wedge\left(x^{\prime}=i\right)$ is independent of $j$ and has to computed only once. It requires $n$ quantifications, $3 n$ Boolean AND's, and $n$ Boolean OR's. The outer computation takes $n$ quantifications (once for each $j$ ), $2 n$ Boolean AND's, and $n$ Boolean OR's. The bounds follow (QED).

Algorithmically, we can think of our algorithm as consisting of two passes over the cycle sets, with the first pass computing $S\left(x^{\prime}, y\right)=\sum_{i}\left(\exists x T(x, y) \wedge c_{i}(x)\right) \wedge \overline{c_{i}(y)} \wedge\left(x^{\prime}=i\right)$. 
I. Let $S(x, y)=0$.

For each cycle set $c_{i}$

1. $U_{i}(y)=\left(\exists x\left(T(x, y) \wedge c_{i}(x)\right)\right) \wedge \overline{c_{i}(y)}$.

2. $S\left(x^{\prime}, y\right)=S\left(x^{\prime}, y\right)+\left(U_{i}(y) \wedge\left(x^{\prime}=i\right)\right)$.

The second pass takes $S\left(x^{\prime}, y\right)$ and returns the faimess graph $R\left(x^{\prime}, y^{\prime}\right)$. The algorithm is as follows.

II. Let $R\left(x^{\prime}, y^{\prime}\right)=0$.

For each cycle set $c_{j}$

1) $U_{j}\left(x^{\prime}\right)=\exists y\left(S\left(x^{\prime}, y\right) \wedge c_{j}(y)\right)$

2) $R\left(x^{\prime}, y^{\prime}\right)=R\left(x^{\prime}, y^{\prime}\right)+\left(U_{j}\left(x^{\prime}\right) \wedge\left(y^{\prime}=j\right)\right)$.

We remark that $U_{i}(y)$ is the set of next states from $c_{i}$ not in $c_{i}$, and $U_{j}\left(x^{\prime}\right)$ is the set of cycle sets that have an edge, not contained entirely in $c_{j}$, to a state in $c_{j}$. We use these observations in the next section.

\subsection{Improving the Structure of the Fairness Graph}

In this section, we describe techniques which give a better model of $Q$ in terms of $Q_{f}$. Consider example a in figure 3 , where $s_{1}, s_{2}, s_{3}, s_{4}$ are the states and $c_{1}, c_{2}, c_{3}, c_{4}$ are the cycle sets. Assume in the first pass we process $c_{1}, c_{2}, c_{3}, c_{4}$ in that order. When processing $c_{4}$, we find that $U_{4}(y)=0$, which means $c_{4}$ has no outgoing edges. We call such cycle sets sink cycle sets. Sink cycle sets cannot be involved in any fair cycles, and can be deleted from $Q$. Hence $s_{4}$ and $c_{4}$ are deleted. Now, $s_{2}$ has no outgoing edges, and can be deleted by the forward stable set operator (recall this operator deletes any states which cannot reach a cycle). Let optimization 1 denote the deletion of sink cycle sets as well as the application of forward and backward stable set operators in the first pass. Optimization 1 in this example deletes $s_{2}, s_{4}$ and $c_{4}$ from $Q$.
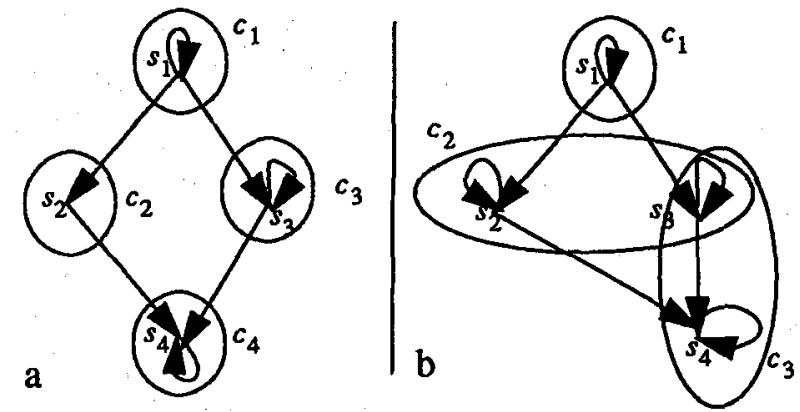

\section{Eigure 3}

Now, consider example $b$ in figure 3. Assume in the first pass $c_{1}, c_{2}, c_{3}$ are processed in order. Since $c_{3}$ is a sink cycle set, states $s_{3}$ and $s_{4}$ are deleted. The stable set operators cannot delete any more states in this case. When we processed $c_{2}$, we added the pair $\left(1, s_{3}\right)$ to $S\left(x^{\prime}, y\right)$, which means $c_{1}$ has an edge to $s_{3}$. But since $s_{3}$ is deleted from the graph, this is not the case any more. The question is whether we will have an edge $(1,2)$ in $R\left(x^{\prime}, y^{\prime}\right)$. We call edges in $R\left(x^{\prime}, y^{\prime}\right)$ which do not exist in the final faimess graph, fake edges. Assume we modified our algorithm in step 1 of pass 2 to $U_{j}\left(x^{\prime}\right)=\exists y\left(S\left(x^{\prime}, y\right) \wedge c_{j}(y) \wedge A(y)\right)$, where $A(y)$ is the current active set of states 
in $Q$. Note that in the first pass, we always restrict $T(x, y)$ to $A(x)$. We also assume that the stable set operators are originally applied to $Q$, before the building of faimess graph is started. We have the following important theorem.

Theorem 2 If optimization 1 is applied during the first pass, then $R\left(x^{\prime}, y^{\prime}\right)$ contains no fake edges.

Proof Let $(u, v)$ be an edge from $c_{i}$ to $c_{j}$ in the original $Q$ (example a, figure 4). Then, $(i, j)$ is fake edge in $R\left(x^{\prime}, y^{\prime}\right)$ iff at least one of $u$ or $v$ is deleted by optimization 1 . We distinguish between three cases.

Case 1. $v$ is deleted by optimization 1 . Then $v \notin c_{j}$ in the second pass, and the pair $\left(i, c_{j}\right)$ gets deleted from $S\left(x^{\prime}, y\right)$ in pass 2 , step 1, and does not gives rise to the edge $(i, j)$ in $R\left(x^{\prime}, y^{\prime}\right)$.

Case 2. Only $u$ is deleted in the first pass, and this occurs because $u$ belongs to some sink cycle set $c_{k}$. We have that $\nu \notin c_{k}$. But, we have an edge from $c_{k}$ to $c_{j}$, i.e. $c_{k}$ is not a sink cycle set. We have reached a contradiction.

Case 3. Only $u$ is deleted in the first pass, and this occurs because of application of a stable set operator after some sink cycle set $c_{k}$ is deleted (example $b$, figure below). Since $v$ is not deleted, $v$ can reach a fair cycle. Hence, $u$ can reach a fair cycle. Thus, $u$ is not deleted by forward stable set operator. So, $u$ is deleted by backward stable set operator. Since, before deletion of $c_{k}, u$ could be reached by some cycle, there is a cycle in $c_{k}$ which can reach $u$. But, since $u \notin c_{k}$, we have that $c_{k}$ has an out-going edge, i.e. it is not a sink cycle set. We have reached a contradiction again (QED).
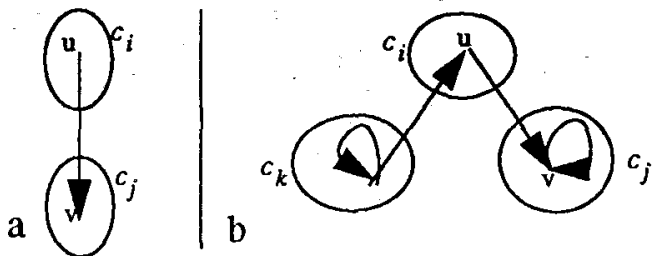

b

\section{Figure 4}

Now, consider the following example. Assume in the second pass, we process $c_{1}, c_{2}, c_{3}$ in that order. When processing $c_{2}$, we find that $U_{2}\left(x^{\prime}\right)=0$, i.e. $c_{2}$ does not have any in-coming edges. We call such cycle sets, which cannot be involved in any fair cycles, source cycle sets. Let optimization 2 be the deletion of source cycle sets and application of the stable set operators in the second pass.

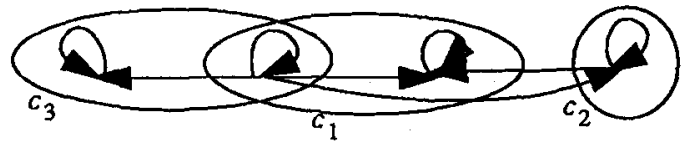

Figure 5

Lemma 7 Applying optimization 2 can result in fake edges.

Proof In figure 5, after $c_{2}$ is processed the edge $(1,2)$ is added to $R\left(x^{\prime}, y^{\prime}\right)$. Then, in processing $c_{3}$, we find that $U_{2}\left(y^{\prime}\right)=0$, so we can delete $c_{3}$. Thus $(1,2)$ is a fake edge (QED).

Consider figure 6. After the faimess graph is built, we notice that $c_{3}$ is not involved in any cycle of the fairness graph. The states of such a cycle set cannot be involved in any fair cycle, and can be deleted from the state graph. Let optimization 3 denote the 
deletion of such cycle sets and the application of the stable set operators, after the fairness graph is built.

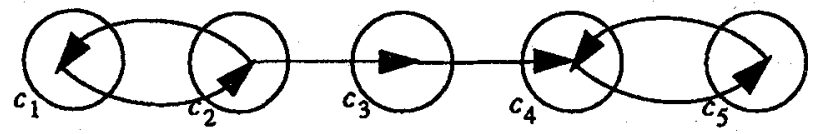

Eiqure 6

Lemma 8 Application of optimization 3 can result in fake edges.

Proof Figure 8 gives an example, where the state graph $Q$ is on the left and the fairness graph $Q_{f}$ on the right. Optimization 3 deletes $c_{2}$. By deleting the nodes of $c_{2}$ from $Q$, the edges $(1,3)$ and $(3,1)$ in $Q_{f}$ become fake (QED).
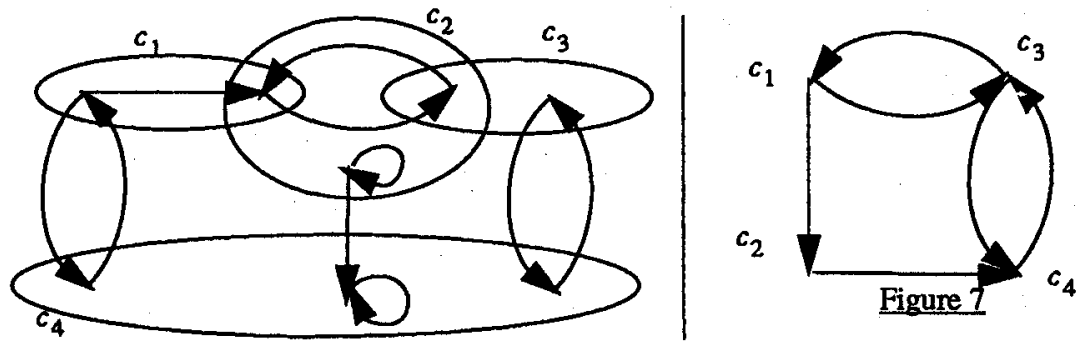

In summary, optimization 1 does not create fake edges, whereas optimizations 2 and 3 might. To deal with fake edges, we have implemented two algorithms. Because we have not run enough examples, we cannot which is preferred at this point. The first algorithm is as follows.

1. Do the first pass with optimization 1.

2. Do the second pass with optimization 2. If any states are deleted, go back to step 1, and restart.

Otherwise, go to step 3.

3. Perform optimization 3 on the faimess graph. If any states are deleted, go back to step 1, and restart the computation.

Note that the first algorithm re-starts the computation as soon as any state is deleted by optimization 2 and 3. The second algorithm is given below.

1. Do the first pass with optimization 1.

2. Do the second pass with optimization 2.

3. Perform optimization 3 on the fairness graph.

4. If any states are deleted in steps 2 or 3, go back to step1.

This algorithm carries out the computation to the end, ignoring possible fake edges. At the end, if there is a possibility of fake edges (because of deletion of states by optimizations 2 or 3), the computation is re-started.

\section{Clustering Cycle Sets}

In this section, we formulate the problem of minimizing the number of fairness constraints. We show that the problem is NP-complete, and propose a heuristic solution.

\subsection{The Clustering Problem}

Definition An acyclic cluster is any sets of vertices in $Q_{f}$ which do not contain a cycle.

Theorem 3 Let $S_{1}, \ldots, S_{p}$ be a partition of vertices of $Q_{f}$ such that every $S_{i}$ is an acyclic cluster. Let $Q^{\prime}$ have the same states as $Q$, but with $p$ cycle sets $c^{\prime}{ }_{1}, \ldots, c_{p}^{\prime}$, where $c^{\prime}{ }_{i}$ is formed by taking the union of all cycle sets in $S_{i}$. Then, a cycle os fair in $Q$ iff it is fair in $Q^{\prime}$.

Proof Let $\Gamma$ be a fair cycle in $Q$. The only way $\Gamma$ is not a fair cycle in $Q^{*}$ is for $\Gamma$ 
to be entirely contained in some $c^{\prime}{ }_{i}$. Assume this is the case. By lemma 3, every shadow of $\Gamma$ is a cycle in $Q_{f}$. Hence the shadow involving cycle sets only in $S_{i}$ is a cycle involving only states in $S_{i}$. But this contradicts the assumption that each $S_{i}$ is an acyclic cluster. Conversely, assume $\Gamma$ is a fair cycle in $Q^{\prime}$. The only way for $\Gamma$ not to be a fair cycle in $Q$ is for $\Gamma$ to be contained entirely in some $c_{j}$. Let's say $c_{j}$ is in some partition $S_{i}$. Since $\Gamma$ is fair in $Q^{\prime}$, it is not entirely contained in $S_{i}$. Hence it cannot be contained in $c_{j}$ (QED).

Note that the above theorem can be used to reduce the number of fairness constraints. We call this technique clustering. The associated optimization problem is to decompose a directed graph $\left(Q_{f}\right)$ into a minimum set of acyclic clusters. This problem is NP-complete, since it is equivalent to the Partition into Forest problem described in [GJ79]. We now show how this problem can be reduced to the decomposition of each strongly connected component (SCC) of $Q_{f}$.

Lemma 9 Let the SCC's of $Q_{f}$ be given. Assume each is decomposed separately into a set of acyclic clusters. The union of a set of acyclic clusters each from different SCC's is again an acyclic cluster.

Proof This follows from the definition of SCC's (QED).

Theorem 4 Assume each SCC of $Q_{f}$ can be decomposed into a minimum of $m_{i}$ acyclic clusters. Let $M=\max \left\{m_{i}\right\}$. Then, $M$ is the solution of the partition into forest problem.

Proof Clearly by lemma 9, a solution with $M$ clusters can be constructed by clusters with at most one element from each SCC. Now assume $M$ is not minium, and let a set of $M^{*}<M$ acyclic clusters $S_{1}, \ldots, S_{M^{\prime}}$ be given. We can built a clustering $W_{1}, \ldots, W_{M}$ for each SCC $W$ with at most $M^{\prime}$ clusters by taking $W_{i}=S_{i} \cap W$. This contradicts that $m_{i}$ is a minimum solution for each SCC (QED).

The above theorem gives us a way of clustering vertices of $Q_{f}$ by processing each SCC separately.

Example Consider the example in Figure 8, which shows a faimess graph with three SCC's: $\{1,2,3\},\{4,5,6\}$, and $\{7,8,9,10\}$. The first SCC can be decomposed into $\{1,(2,3)\}$, the second into $\{4,(5,6)\}$, the third into $\{(7,8),(9,10)\}$. Thus, $M=2$. The final decomposition is $\{(1,4,7,8),(2,3,5,6,9,10)\}$, and we have reduced the cycle sets from 10 to 2 .

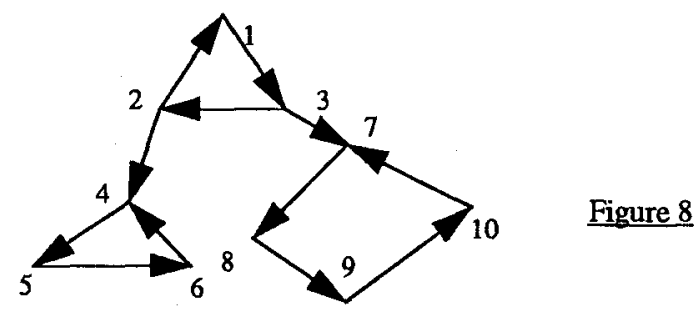

5.2 Heuristic Clustering

We use a simple recursive, greedy heuristic for the clustering of each SCC, which is described below. 
1. If two vertices do not form a cycle, merge them into a hyper node.

2. If any merges were performed in step 1, call the routine recursively on the graph formed by taking each cluster as a node.

Note that in step 1 , a vertex is merged with at most one other vertex. This allows many clusters to grow concurrently (as opposed of starting with one and continuously adding to it). In step 2, if any clustering was done in step 1, we form a new graph by taking the clusters found in step 1 as new vertices and putting an edge between them if the vertices in the cluster were connected in the original graph.

Remark We need to compare our heuristic to the heuristic of maximally growing a cluster until no more nodes can be added, and then continuing with the next cluster. We also propose to process the SCC's in decreasing size. Since, we would not need to decompose a small SCC into less than $M=\max \left\{m_{i}\right\}$ seen so far, we can use this as an early bounding step to speed up processing.

\section{Experiments}

We have recently implemented our algorithms in HSIS, a formal verification system developed at UC Berkeley that supports language containment and model checking using BDD's. To test our algorithms, we used an abstracted description of a SUN Sparc memory model [Sparc-8], [DNP93]. Our system model is a memory system that is accessed by a number of processors, as in a typical shared memory multiprocessor system. The memory is modeled abstractly as a single port device that grants write access to the attached processors by randomly selecting one.

For our experiments we mapped an assembly code sequence (from [Sparc-8]) that implements spin locking to an automaton representing a processor. Another automaton models the memory and its random scheduling. To model general computation, both the automata for the processor and the memory have several states with self loops: the processor can stay in an idle state indefinitely long before attempting to acquire the lock; it can also wait indefinitely long returming to its computation loop, and stay in its critical section (after acquiring the lock) for an unspecified period of time. Each of these states becomes a one-element cycle set, since we want to exclude the behavior from the system where processors remain in one state forever. Similarly, we require that the memory does not always choose to service the same processor, i.e. the memory is "fair".

We verify the following liveness property: a processor will eventually be able to acquire the lock (no starvation). We verified the property for systems with 3 and 4 processors. The results are summarized in table 1 . All times are in seconds and are measure on a DECsystem 5000/260 machine with $128 \mathrm{MB}$ of memory.

Table 1:

\begin{tabular}{c|c|c}
\hline num of processors & 3 & 4 \\
\hline reachable states num & 10685 & 143228 \\
\hline reachability & 5.35 & 116.7 \\
\hline main comp. w/o f.g. & 5.47 & 61.45 \\
\hline total time w/o f.g. & 15.10 & 221.98 \\
\hline building the f.g & 1.45 & 14.81 \\
\hline main comp. with f.g & 0.0 & 0.0 \\
\hline total time with f.g. & 11.11 & 175.1 \\
\hline improvement factor & 3.77 & 4.15 \\
\hline
\end{tabular}


Note that in this example, we were able to decide the language containment check by only analyzing the faimess graph. Hence, the main computation time with the fairness graph analysis is 0 . The last row is obtained by taking the time of main computation without our technique, divided by the sum of building the faimess graph and the time for the main computation with our technique.

\section{Conclusions and Future Work}

Verification using language containment involves performing reachability, and then calling an algorithm whose running-time is proportional to the number of fairness constraints (called the main computation). If the number of faimess constraints is large, the main computation can be time-consuming. Our goal is to make verification using language containment basically as efficient as performing reachability. This work investigated ways to make use of the information inherent in the graph induced by the faimess constraints on a system, the fairness graph, to reduce the run-time of the main computation. We suggested techniques to construct and process the fairness graph. Our techniques are a useful complement to early failure detection, because they can help when a property passes. We used the example of an abstract memory model as a test-case to validate the techniques, and concluded that the methods hold promise. On this example, we observed a factor of 4 reduction in the run-time of the main computation. Because our implementation is preliminary, there may also be some scope to improve the efficiency of computing the faimess graph and the clustering.

\section{References}

[Bry86] R. E. Bryant, "Graph Based Algorithms for Boolean Function Manipulation", IEEE Trans. on Computers, C-35(8):677-691, August 1986.

[CES86] E. M. Clarke, E. A. Emerson, and A. P. Sistla. "Automatic Verification of FiniteState Concurrent Systems Using Temporal Logic Specifications", ACM Transactions on Programming Languages and Systems. 8(2), pp.244-263, 1986.

[DNP93] D. L. Dill, A. G. Nowatzyk, S. Park, "Formal Specification and Verification of Abstract Memory Models", Conference on Hardware Description Languages, 1993.

[HTKB92] Ramin Hojati, Herve Touati, Robert P. Kurshan, Robert K. Brayton, "Efficient $\omega$ Regular Language Containment", Computer-Aided Verification, 1992.

[HSB94] Ramin Hojati, Vigyan Singhal, Robert Brayton, "Edge-Streett/Edge-Rabin Automata Environment for Formal Verification Using Language Containment", UCB/ERL Tech. Report, M94/12, 1994.

[Kur87] R.P. Kurshan, "Reducibility in Analysis of Coordination", In Discrete Event Systems: Models and Applications, volume 103 of LINCS, pages 19-39, 1987.

[GJ79] Michael R Garey, David S. Johnson, "Computers and Intractability, A Guide to the Theory of NP-Completeness", Freeman and Co., 1979

[Sparc-8] The SPARC Architecture Manual, Version 8, SPARC International Inc., Menlo Park, CA, 1992.

[Tou90] H. Touati, H. Savoj, B. Lin, R. K. Brayton, A. S. Vincentelli, "Implicit State Enumeration of Finite State Machines Using BDD's", International Conference on Computer-Aided Design, 1990.

[VW86] M.Y. Vardi and P.L. Wolper, "An Automata-Theoretic Approach to Program Verification", Logic in Computer Science, pages 332-334, 1986 